Удк: 81'276.6:574

DOI: 10.22363/2313-2299-2018-9-2-302-319

\title{
STRUCTURE OF COGNITIVE TYPE OF SCIENTIFIC ARTICLE ON MOUNTAIN ECOSYSTEMS
}

\author{
Sofiya N. Semenova \\ Kuban State University \\ Stavropolskaya st., 149, Krasnodar, Russia, 350040
}

\begin{abstract}
The structure of cognitive type of scientific article on mountain ecosystems is given in the article. The purpose of the research is to build a structure of cognitive type of scientific article describing mountain ecosystems. For achievement of the stated work's aim it was necessary to solve the following problems: 1) to explore the text corpus of scientific article on mountain ecosystems; 2) to define the topical structure of cognitive type of scientific article describing mountain ecosystems; 3 ) to present verbal fragments for each compositional-thematic unit; 4) to describe pragmatics of text realization of scientific article on mountain ecosystems; 5) to build mini-thesaurus of words and phrases. The author justifies the thesis that the cognitive type of a scientific article is the basic part of one of the cognitive blocks in the system of human knowledge representation in subject area "Mountain ecosystems". The main elements as: (people's life's work, environmental pollution, environmental protection, constant nature monitoring) are defined. There are words and phrases which are arranged in sub-topics in English and Russian. These words and phrases are samples from the text corpus of the subject area "Mountain ecosystems" and are not ready texts, but their blank for discoursive activity not only for specialists in this sphere but for everybody who is interested in ecological problems in English and Russian. Summing all up, it can be noted that the structure of the cognitive type of the scientific articles is a mental-linguistic frame used by the authors of the scientific articles in the process of generation of texts and the readers in the process of understanding. The structure of the cognitive type is presented by a scan of a specific topic and verbal fragments for each thematic unit. The reader is able to solve or suggest a solution to the critical environmental situation in the ecosystem based on the data obtained in the course of the text description of the environmental problems of mountain ecosystems.
\end{abstract}

Key words: structure of cognitive type; subject area "Mountain ecosystems"; text corpus; linguistic frame; representation of verbal fragments; discursive activity; knowledge; cognitive activity; pragmatics of textual implementations; thesaurus

\section{INTRODUCTION}

When studying texts of a certain subject area a cognitive type (the concept given by Baranov A.G.) is introduced into the study as a mental-linguistic frame for finding the prototypical meanings of the cultural world on the basis of cognitive knowledge $[1 ; 2]$.

The purpose of this work is to build a structure of cognitive type of scientific article on mountain ecosystems. We think that it is a macrostructure, which in its turn consists of a number of microstructures demonstrating the detailed distribution of data in the subject area "Mountain ecosystems". 
To achieve the goal fixed in the work, it was necessary to solve the following problems:

1) to study the corpus of texts of scientific article describing mountain ecosystems;

2) to determine the thematic structure of the cognitive type of scientific article characterizing mountain ecosystems;

3) to present verbal fragments to each composition-thematic block;

4) to describe the pragmatics of textual implementations of scientific article on mountain ecosystems.

Our survey of scientific works on the following linguistic problems are based on the Russian and foreign researchers' studies, dealing with:

1) thesaurus $[6 ; 18 ; 20 ; 30]$;

2) genre $[3 ; 9 ; 17 ; 27]$;

3) $\operatorname{text}[11 ; 14 ; 22 ; 25]$;

4) discourse $[4 ; 6 ; 10 ; 13 ; 16 ; 23 ; 26 ; 29]$;

5) cognitive linguistics $[5 ; 12 ; 15 ; 19 ; 21 ; 24 ; 28]$.

The cognitive type of a scientific article describing the ecological problems of mountain ecosystems is the main part of one of the cognitive blocks "Mountain ecosystems" in the system of the representation of human knowledge in the subject area "Mountain ecosystems". The structure of cognitive types consists of a complex of objective elements that represent various information concerning the ecology of mountain ecosystems. As a result of the analysis of more than 200 descriptions of the ecological situation in the mountains, the following structure of the cognitive type of scientific article was obtained (1-7) (High Priorities. GEF's Contribution to Preserving and Sustaining Mountain Ecosystems. Washington, 2002. Vol. 13. № 1. 2002. 30 p.; Meith N. World Conservation: Mountain High. Switzerland, 2002. Vol. 33. № 1. 40 p; Mountain Momentum: Agenda for Today and Policy beyond IYM 2002. The United Nations University. 2002 International Year of Mountains. 2002. 26 p.; Nemolyaeva L. Ensuring Long-Term Conservation of the Altai-Sayan Ecoregion WWF Project: Achievements and Lessons Learned. M., 2001. 16 p.; Russian Bird Conservation Union. M., 1998. 11 p.; Supporting Environmental Cooperation in Central Asia. Philippines, 2002. 49 p.; Tropical Montane Cloud Forests: Time for Action. Switzerland, 1999. 29 p.)

\section{THE THEMATIC STRUCTURE OF THE COGNITIVE TYPE OF SCIENTIFIC ARTICLE DESCRIBING ECOLOGICAL PROBLEMS OF MOUNTAIN ECOSYSTEMS}

\section{People:}

1) People's lives; 2) conflicts.

2. Factors affecting biodiversity change:

1) Global climate change; 2) reduction of forest; 3 ) overgrazing of livestock;

4) mining industry.

3. Monitoring of mountain ecosystems:

1) hydrology; 2) landscapes; 3) flora; 4) fauna.

4. Pollution and environmental degradation of Mountain ecosystems.

5. Protection of the environment of mountain ecosystems:

1) ecotourism; 2) resources: soil, water, forest. 


\title{
6. International organizations for the protection of the environment of moun- tain ecosystems:
}

1) The name; 2) the purposes of the organizations; 3) financing; 4) the results.

As a result of the study of the texts' corpus, we came to the conclusion that the thematic structure of the cognitive type of scientific article describing the ecological problems of mountain ecosystems consists of informative factors combined with subordinate and co-ordinate textual and classification features. In our work a number of words and phrases are presented. The given examples are arranged in sub-topics in English and Russian. These words and phrases serve as a sample of the corpus of texts of the subject area "Mountain ecosystems" and are not ready-made texts, but they are preparations for the discursive activity not only for specialists in this field, but for all those who are interested in environmental problems of mountain ecosystems in English and Russian.

\section{REPRESENTATION OF VERBAL FRAGMENTS}

\author{
1. People \\ People's life's work
}

Hunting / poaching (охота), aggregation (собирание), cutting (вырубка), perforation / boring / drilling (бурение), осcupancy (заселение), horticulture (садоводство), husbandry (сельское хозяйство), melioration (мелиорация), recultivation (возделывание).

\section{Conflicts}

To plague mountain region (насылать бедствие на горный регион); war disaster (военное бедствие), armed combat in mountain region (вооруженный бой в горном регионе), widespread conflicts in mountain regions (распространенные конфликты в горных регионах).

\section{Factors affecting biodiversity change}

Global climate change

To be of vital importance (быть жизненно необходимым), glaciers (ледники), to affect the lives of mountain people (влиять на жизнь людей, живущих в горах).

\section{Deforestation}

To degrade natural forest ecosystems (подвергать деградации природные лесные экосистемы), to disappear rapidly (быстро исчезать), to be for legal and illegal drug production (быть для законного и незаконного производства лекарств).

\section{Overgrazing}

To drive livestock to mountains (вести домашний скот к горам), to cause soil erosion and desertification (привести к эрозии почвы и опустыниванию), grazing land for cattle (пастбище для скота). 


\section{Mining Industry}

To constitute significant threat (представлять значительную угрозу), construction of new railroads (строительство новых железных дорог), dams (дамбы), hydroelectric power plants (гидроэлектрические предприятия).

\section{Monitoring of mountain ecosystems}

\section{Hydrology}

To supply major rivers in the world (питать основные реки в мире), to quench thirst from mountain water (утолять жажду горной водой), to experience chronic water shortages (переживать постоянную нехватку воды).

\section{Landscapes}

To prevent soil erosion (предотвратить эрозию почвы), to be common (быть обычным), to be sensitive to climate changes (быть чувствительным к климатическим изменениям).

\section{Flora}

Concentration of flora (концентрация растительности), tree (дерево), shrub (куст), flower (цветок), grass (трава), rare plants (редкие растения).

Fauna

Animal kingdom (царство животных), beast (зверь), male (самец), female (самка), mammal (млекопитающее), bird (птица), reptile (рептилия), rodent (грызун), fish (рыба), worm (червь), insect (насекомое), predator / prey (хищник).

\section{Pollution and environmental degradation of mountain ecosystems}

Burning of fossil fuels (сгорание ископаемых горючих), pollution from mining operation (загрязнение от добывающих работ), water pollution (загрязнение воды).

\section{Protection of mountain ecosystems' environment}

\section{Ecotourism}

To include incentives to conserve ecosystems (включать стимулы, чтобы coхранить экосистемы), to conserve biodiversity (сохранять биоразнообразие), to conserve the area's unique natural heritage (охранять территории уникального природного наследия).

\section{Resources}

\section{Soil}

To use vegetation to minimize surface erosion (использовать растительность для того, чтобы уменьшить поверхностную эрозию), control of soil erosion (контроль за эрозией почвы), proper drainage (необходимый дренаж).

Water

To improve flood forecasting (усовершенствовать прогноз наводнения), to improve water quality (улучшить качество воды), water conservation (сохранение воды). 


\section{Forest}

To protect crops from strong winds (защищать урожаи от сильных ветров), management of forest resources (управление лесными ресурсами).

\section{International organizations for the protection of mountain ecosystems' environment}

\section{Names}

ACP (Agricultural Conservation Programme) - Программа помощи фермерам в сохранении почвенных, водных и лесных ресурсов, EEF (European Environmental Foundation) - Европейский фонд окружающей среды, IAWPR (International Association on Water Pollution Research) - Международная ассоциация по исследованию загрязнения воды, NEAP (National Environmental Action Programme) Национальная программа действий по охране окружающей среды, WWW (World Weather Watch) — Всемирная служба погоды.

\section{The aims of the organizations}

To contribute to the maintenance of productive lands (способствовать поддержанию продуктивности земель), to provide a source of new medications and chemical substances (предоставлять источник новых лекарственных и химических веществ).

\section{Finance}

Private company financing (финансирование частной компанией), a group financing (финансирование группой), agency financing (финансирование агентством), government financing (финансирование правительственном), money (деньги), dollars (доллары).

Results

To support development of networks (поддерживать развитие сетей), ecotourism (экотуризм), network (сеть), involvement in mountains (вмешательство в горы), conservation strategies (стратегии защиты), establishment of new protected areas (фиксирование новых охраняемых территорий).

\section{ORIGINAL ENGLISH EXCERPTS FROM SCIENTIFIC ARTICLES AND RUSSIAN TRANSLATION}

In the following excerpts from the scientific articles, we tried to illustrate ecological problems that resulted from irrational use of mountain ecosystems by humans. The presented translations after each excerpt of the scientific article are made by us.

"Climate change is now accepted as a reality by most scientists and by a significant proportion of policy-makers in governments around the world. Since mountains cover nearly a quarter of the Earth's land surface, climate change is clearly of concern to the hundreds of millions who live in mountain area. Yet climate change in mountains is of vital importance to the entire global population" (Meith N. World Conservation: Mountain High. Switzerland, 2002. Vol. 33. № 1. P. 14).

(В настоящее время изменение климата понимается большинством ученых и высших чиновников в правительствах во всем мире как действительность. Поскольку 
горы занимают почти четверть всей поверхности Земли, изменение климата беспокоит сотни миллионов людей, живущих в горных областях. Изменение климата в горах имеет огромное жизненное значение и для всего мирового сообщества.)

From the above passage it follows that humanity aspires to live comfortably. Therefore it uses all the natural resources of mountain ecosystems for its material good. All this is done without rational nature management in favor of humanity, however to the detriment of the mountain ecosystem. In fact, damage will be done only to future generations of mankind. So, in this passage, the author of the scientific article implicitly calls on all to protect and use natural resources correctly in order to preserve them for future generations.

"The consequences will have the most dramatic impact on the lifestyles of the indigenous people, animal migration routes and species composition. It will also increase the vulnerability of forest ecosystems to forest fires and pests. Climate change impact already been vividly traced in the region, such as the anomalous floods in Tyva in spring 2001, shrinking of glaciers, winter warming, changes of forest species at the top forest boundary, and changes in vegetation types on several slopes" (Nemolyaeva L. Ensuring Long-Term Conservation of the Altai-Sayan Ecoregion WWF Project: Achievements and Lessons Learned. M., 2001. Р. 6).

(Последствия будут иметь самое драматическое воздействие на образ жизни местных жителей, маршруты миграции животных и видовой состав. Это также увеличит уязвимость лесных экосистем к лесным пожарам и вредителям. Результаты изменения климата уже прослеживаются в регионе, например, аномальные наводнения в Туве весной 2001 года, сокращение ледников, зимнее потепление, изменение лесных видов на наивысшей границе леса и изменения видов растительности на некоторых склонах.)

As we can see, from this excerpt from the scientific article it follows that climate change in mountain ecosystems will entail:

1) the disappearance of many species of animals and plants;

2) temperature change;

3) change in atmospheric pressure;

4) change in the amount of precipitation;

5) deterioration of water quality;

6) change in air composition.

All these factors must be harmoniously linked with each other. Even the slightest increase or decrease in temperature by $1^{\circ} \mathrm{C}$ can affect the entire nature of the mountains. Therefore, it is necessary to conduct scientific monitoring of all components of mountain ecosystems regularly in order to protect the environment.

"Climate change is undoubtedly taking place, and mountain ecosystems and the people who depend on them will be directly affected. At the same time, mountains are also key locations for understanding what is happening to our world. Monitoring and research focusing on mountain glaciers, hydrological systems, and sensitive species are of vital importance to us all" (Nemolyaeva L. Ensuring Long-Term Conservation of the Altai-Sayan Ecoregion WWF Project: Achievements and Lessons Learned. M., 2001. P. 6).

(Изменение климата, несомненно, имеет место. Оно коснется и горных экосистем, и людей, которые зависят от них. В то же время горы - это ключ для понимания 
того, что происходит с нашим миром. Контроль и исследование состояния сред, сосредотачивающиеся на горных ледниках, гидрологических системах, уязвимых видах, имеют жизненно важное значение для всех нас.)

In this passage from the scientific article the author states the problems of mountain ecosystems associated with global climate change. The author notes that you need to monitor and investigate all components of mountain ecosystems.

As it can be seen from the above passages, the main topic of scientific articles lies in environmental issues and environment of mountain ecosystems [7. P. 56]. The main elements (human activity, environmental pollution, environmental protection, continuous monitoring) show in detail the environmental situation in the mountain regions. Almost all scientific articles provide a detailed description of the problem that characterizes the texts as descriptive, as they were in the past, are in the present and will give forecast for the future. In the process of discourse the author of the scientific article meticulously describes the details related to all environmental problems, and the reader, interpreting, comes to a conclusion (depending on his personal view of the world picture) [8. P. 145].

With the help of common elements in the meaning and use of units, classes are combined in a field. With the help of specific differences they confront each other as classes of units of one field. All received information is presented in the following mini-thesaurus structured according to the principle of a thematic dictionary, in which lexical filling of the topic blocks takes place. We hope that this mini-thesaurus will serve as a basis for discursive activity.

\section{MINI-THESAURUS}

\section{Ecology (экология)}

Ecocide - экоиид (преднамеренное губительное воздействие на окружающую среду), есо-city - экогород (теоретический образец города будущего, в котором все бытовые и производственные процессы будут осуществляться по безотходному циклическому методу), ecoclimate - экоклимат (климатические условия, непосредственно окружающие особь), ecoclimax — экоклимакс (градиент экосистемы), ecocline - экоклин, градиент экосистемьл (клин адаптации вида или сообщества к градиенту условий среды), ecodemography - экодемография (экология народонаселения), ecodevelopment - экоразвитие, экологически приемлемое развитие (которое наносит наименьшее негативное воздействие окружающей среде), engineering ecology - инженерная экология (научно-прикладная отрасль по созданию природно-технических геосистем и управлению ими, не нарушая механизмов саморегуляции объектов и процессов биосферы), environmental ecology - экологические аспекты охраны окружающей среды, evolutionary eco$\operatorname{logy}$ - эволюционная экология (раздел науки, изучающий изменения, обусловленные развитием жизни на планете), fire ecology — экология пожаров, freshwater ecology - экология пресных водоемов, general ecology — общая экология, global ecology - глобальная (планетарная) экология (экология в приложении к биосфере земли), grassland ecology - луговедение, landscape ecology - ландмафтная эко- 
логия (изучение и сохранение уникальных природных ландшафтов), plant ecologу — экология растений, population ecology — популяционная экология (изучение совокупности популяций одного вида, объединенных общей территорией и генофондом), radiation ecology — радиационная экология, range ecology - экология nастбищ, restoration ecology - восстановительная экология, social ecology сочиальная экология (отрасль, изучающая взаимоотношения социальных групп общества со средой их жизни), stream ecology - экология реки, systemic ecology системная экология, technical ecology - техническая экология (раздел экологии, изучающий влияние техники на экосистемы), terrestrial ecology — экология земных организмов, toleration ecology — «терпимая» экология (допускающая отклонения от стандартных требований), woodland ecology - экология леса, ecospecies эковид (экологический вид, отличающийся от другого по различной приспособленности к условиям обитания), ecosphere - экосфера (совокупность всего живого на Земле во взаимодействии с атмосферой, гидросферой и литосферой), ecostandard - экологические нормы (экостандарт), ecostress - экологический стресс, ecosystem - экосистема (основная экологическая система, включающая флору и фауну в их взаимодействии с окружающей средой), agricultural ecosystem сельскохозяйственная экосистема, alpine ecosystems - альпийские (высокогорные) экосистемь, anolog(ue) ecosystem - искусственно созданная модель природной экосистемы, anthropocentric ecosystem — экосистема (подчиненная интересам человека), aquatic ecosystem - водная экосистема, elastic ecosystem - упругая экосистема (способная к самовосстановлению), endangered ecosystem - экосистема (находящаяся в опасности), ecotype - экотип (генетическая вариация вида, адаптировавшегося к определенной окружающей среде).

\section{Factors Influencing the Ecology of Mountain Ecosystems (факторы, влияющие на экологию горных экосистем)}

Fire - пожар, disease - болезнь, insect attacks - нашествия насекомых, parasitism - паразитизм, ехіstence - присутствие, habitat destruction - разрушение естественной среды растения или животного, population - популяция, evolutionary heritage - эволючионное наследство, genetically distinct - генетически определенныци, protection - защита, liquidation - ликвидация, abolition уничтожение, maintenance - сохранение, adjustment - регулирование, afforestation - облесение, лесонасаждение, agroceonosis - агроценоз, appeasement утоление, mutualism - взаимность, aridity — засуиливость, бесплодность, biodiversity - биологическое разнообразие; palaeodemecology - палеодемэкология, biogeocoenosis - биогеоценоз, palaeoecology — палеоэкология, palaesynecology палеосинэкология, catasrophism - катострофизм, phytocoenosis - фитоиеноз, commensalisms - сокращение, community - община, pollutant - загрязнитель, pollution $=$ contamination - загрязнение, conservancy - охрана (природы), presuppression - подавление, contribution - пожертвование, prohibition - запрещение, cooperation - объединение, protection - защита, reotocation - возобновление, restoration - воспроизводство, salvation - спасение, избавление, environmentalism - экологизм, starvation — голодание, symbiosis — симбиоз, thanatocoenosis - 
общая смерть, fertility — плодородие, weakness — слабость, endemic — эндемик, autecology - аутэкология (раздел экологии, изучающий действия различных факторов внешней среды на отдельные популяции).

\section{General Geology (общая геология)}

Mountain chain - горная цеепь, range - цень, subrange - подуровень, highland — возвыленность, rock — скала, порода (adj. sedimentary — осадочная , igneous $\sim$ — вулканического происхождения $\sim$, metamorphic $\sim$ —метаморфный $\sim$; molten $\sim$ — расплавленная $\sim$, soft $\sim$ — мягкая $\sim($ ant. hard $\sim$ - твердая $\sim)$, erosionresistant $\sim$ — устойчивая от коррозий $\sim$, slate $\sim$ — сланцевая $\sim$, quartzite $\sim$ - квариевая , meta-sedimentary — метаосадочная , granite-gneiss — гранитно-гнейсовая; basement $\sim$ — подвальная $\sim$, granitic $\sim$ — гранитная $\sim$, dominant $\sim$ — доминантная $\sim$, visible — видимая $\sim$ ) — (the Kaibab Limestone, Toroweap Formation, Coconino Sandstone, Hermit Shale, Supai Group, Redwall Limestone, Vmuav Limestone, Bright Angel Shale, Tapeats Sandstone), canyon - каньон, plateaus - плато (the Kaibab, Kanab, Uinkaret, Shivwits), cliffs - yтесы, dams - плотины водохранилища, basin бассейн, debris - обломки, slopes - склонь, gorge - ущелье, rapids - речньле пороги, blocks - блоки; erosion - эрозия, structure - структура, sediment - осадок, weakness - слабость, fossils - останки, the earth's surface поверхность земли, hardness — твердость (ant. softness — мягкость), slippage - обвал, earthquake - землетрясение, sedimentation - отложение осадка, bed - ложе, karst - карст, origin — происхождение, location - расположение, distance - расстояние, glaciation - обледенение, kaolinozation $=$ kaolinisation разрушение гранита, kratogen - устойчивые блоки, lithology = sedimentology наука об осадочных породах.

\section{General Geography (общая география)}

Cosmography — описание мира, position - месторасположение, co-ordinate — координата, latitude — широта, longitude — долгота, layering — расслоенность, length - длина, meridian — меридиан, amplitude — амплитуда, azimuth - азимут, direction of dip - азимут падения, azonality - азональность, zonality - зональность, zonation - зональное расположение, differentiation поясность, Tropic - тропический пояс, tropicality - тропичность.

\subsection{Climate (климат)}

Synoptics - наука о предсказывании погоды, microclimatology - микроклиматология, mesoclimatology - мезоклиматология, ecoclimate — экоклимат, air masses - воздушные массы, humidity = moisture — влажность (adj. summer - летняя, winter - зимняя, spring - весенняя, autumn - осенняя), climatic patterns - климатические характеристики, moist air - влажный воздух, weather — погода (adj. rainy — дождливая $\sim$, snowy $\sim$ - снежная $\sim$, windy $\sim$ ветреная $\sim$, colder $\sim$ - холоднее $\sim$ (ant. warmer $\sim$ теплее $\sim)$, sunny $\sim$ солнечная, sunly $=$ dry $\sim$ - сухая $\sim$ (ant. wet $\sim$ — мокрая $\sim)$, regional $\sim$ - местная $\sim$, 
clear — ясная $\sim$, сусlе — погодный ичикл), storm - шторм (adj. frontal фронтальный $\sim$ ), pressure — давление (adj. high - высокое (ant. low — низкое) ), temperature - температура (high - высокая , low - низкая , average $\sim$ - средняя $\sim$, normal $\sim$ — нормальная $\sim, \sim$ variation between day and night - изменение между днем и ночью, large - большая $\sim$, seasonal — временная $\sim$, freezing - замораживающяая $\sim$, ovenlike — жаркая $\sim$, frostbite $\sim$ обмораживающая ), warming - согревающий (ant. cooling — охлаждающий),

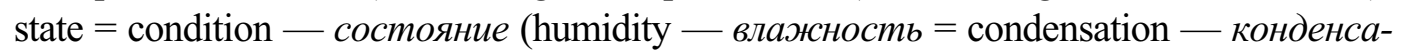
ция, activity - деятельность, aridity — засушливьій = xerophytic - ксерофитньй).

\subsection{Precipitations (осадки)}

Rain — дождь (drizzling - моросящий $\sim)$, rainfall - осадки, shower -ливень, thundershower - ливень с грозой, hail - град, snow - снег, dew - роса.

\subsection{Types of relief (виды рельефа)}

Vertical = altitudal — вертикальный (ant. horizontal — горизонтальный), anaglyphic — рельефный, indicating — обозначающий, indined - наклонный, inevitable — неизбежный, infilling - грядоподобный, ingressive - ингрессионныци, inherited - унаследованный, installed - проектныци, insular - островной, interdunal — междюнный, intermediate - промежуточный, intermixed - смешиваюшийся, internal - внутренний, intractable - неподатливый, intrazonal межзональный, intrusive - интрузивный, invalidated - неполноченный, inverted - обращенный, isobaric - изобарический, isoclinal - изоклинальный, isostatic - изостатический, karstic - карстовый, kataclastic — катакластические структуры, lateritic — латеритический, linear = lining — линейныци, lithologic - литологический, U-shaped - у-образный, lithospheric - литосферньй, lobed - падающиий, longish - длинноватылй, longitudal dunes - барханнылй гряды, absorbed - поглощенныци, absorbing — поглотительный, magmatic - магматический, zenithal - зенитньй, accumulative = aggradational — намыльы platform - активированная платформа, mammillated surface - пузырчатая поверхность; aggraded plains — аккумулятивные равнины, minicontinental миниконтинентальный, minimal - минимальный, Appalachian - аппалачский, archaic — устаревшиц̆, arenaceous — paсcыпчатый, песчанистый, neritic неритовыц̆, argillaceous — глинистый, armoured landform — бронированный рельеф, non-gravity landform - восходящчий рельеф, noticeable - заметный, attractive - привлекательный, original = initial — первичный, beaded - четковиднылй, bedded - наброшеннылй, percolating - инфильтрационныли, centriclinal центриклинальный, rolling - волнистый, sculptured relief — скульптурный рельеф, diverging — расходящииися, separated $=$ divided — отделенный, diversion — водоотводнылй, structural - структурный, stubby - выровненный, subaerial - наземнылй, transversal - поперечныц undulating - холмистый, unimpaired - неповрежденныци, uninverted relief необращенный рельеф. 


\subsection{Mountain composition (состав гор)}

Buttress - выдаюшаяся вперед часть холма или горы, divide - раздел, хребет, versant - склон, metal — щебень, monolith — отдельный каменный блок, monadrock - гора-свидетель = останеи выветривания, zeugen - скала-свидетель, blaen — верховье, acclivity = declivity — склон, authigenous - частиць пород, возникиие в результате кристаллизации, cuvette — впадина, demopleth глубокая впадина между холмами, depression — впадина (ant. sublimity — возвьшенность), ruggedness - неровность, sediment - отложение, slickenside - зеркало скольжения, slippage - обвал, elongation - удаление, stratum - слой, отложение, texture - структура, tumulus - могильный холм, курган, grade уклон, покатость, batholith - гранитный массив в горах.

\subsection{Mountain description (описание гор)}

Mountainous - горныцй, orogenic $=$ orogenetic - горообразуюший, orographical — орографический, available - относительная высота, plaintive - nечальнылй (о горах), banding — полосчатый, passage — горный проход, pedestal — остаточная гльба пород, conformable - параллельное залегание слоев друг над другом, disgeogenous - устойчивыле с трудом разрушаемые породы, escarpment крутой откос, synclinorium - сложная складчатая структура, heteroclinal гетероклинальная складка, impermeable = impervious rocks - водоупорные горные nороды, impressive rocks - сильныле породы, incised - врезанный, industrial stones - поделочные камни, injected = injenction - инъекиионньй $=$ обломочньй, melanocratic rocks - мелократовые горные породы, aleuritic - алеуритовый, mesotrophic - мезотрофический, alkalitrophic — щелочнотрофический, metallogenic - указывающий на происхождение железных руд, metamorphic — метофморфический, altiplanation terrace - нагорная терраса, amygdaloidal structure миндалекаменная текстура, molten — расплавленньй, antipodal — расположенньій строго друг против друга, moving - движущииися (ant. motionless - неподвижный), archeological — археологический, orocratic — горосозидательный, basaltic базальтовый, — палеотипные породы, canotypal rocks - кайнотипныле породы, cardinal - главный, cavernous пещеристый, pelitic - пелитовый - обломочное отложение, penecontemporaneous folding - конседиментационная складчатость, cheesewring - грибообразная скала - останец выветривания, petrographic изучающий горныле породы, poikilitic structure - пойкилитовая структура, pointed - остроконечныли, conglomerate - неоднородная горная порода, polygenetical mountains - полигенетические горы, porous mountain - nористая гора, porphyritic - неравномерно-зернистая структура магматических горных пород, posthumous folding - постумная складчатость, conveуing - водопропускной, protruding - вылвинутылй, psephitic - крупнообломочныц pyramidal peak — остроконечная пирамидальная горная вершина, covered mountain - покрытая гора, crenulate - зубчатый, crooked - согнутыли, cryptoclastic rock - крытообломочная горная порода, dangling - качающийся, degraded mountains - остаточные горы = residual — остаточная порода, denudational slope — денудационный склон, rudaceous - грубообломочный, detrial rocks - 
обломочные горные породы, sandblasted pebble - обломок горной породы - трехгранник, siliceous rocks - кислье горныле породы, epiplatformian mountains - возрожденные горы, ехоgenous - экзогенныле породы, taxitic structure - такситовая структура горных пород, fissured - треснутылй, fixed - закрепленный, toothed зубчатый, foliated structure - листовая структура, tremendous - огромный, fragmental texture - кластическая структура, ultrabasic rocks - ультраосновные горныле породы, furrowed - морщчинистый, unfossiliferous rocks - «немыле» горныле породы, gate — горный проход.

\subsection{Mountain Processes (горные процессы)}

Orogenesis - горообразование, orogeny - складчатость, humidification увлаждение, icing - ледообразование, illuviation — вмывание, imbrication - перекрытие внахлестку - по типу укладки черепицы, impoverishment - обеднение, impression - оставленное на поверхности мягкое отложение - песок, incision углубление рекой своего русла, inclusion - включение обломков древней горной nороды, indentification - выдалбвливание, induration - окончание, industrialization - индустриализация, insolation - освещение солнечными лучами, intention - намерение, intergration - объединение, interpretation - объяснение, introduction - ознакомление, inversion - изменение, katothermy - катотермия, lapiesation - каррообразование, location - размещение, ablation - выветривание горной породы под воздействием воды, abolition - уничтожение, abrasion - истирание, abstraction - отделение, magmatism - магматизм, magnetism - магнетизм, accentuation - обострение, acclimatization - акклиматизация, manipulation - манипуляция, map assemblage - компановка карты, adjustment - уравновешивание, meandering - изгибы, agglomeration - процесс повышения поверхности, aggradation - расширение площади распространения, metamorphism - превращение древних пород в новые, migration = movement - движение, mineralization - минерализация, altimetric — распределение высот, altiplanation процесс выравнивания в высокогорных областях, anatexis - процесс расплавления горных пород, mountain building - горостроение, appearance - появление, nivation - разрушающее воздействие мороза на породы вблизи тающих снегов, backfolding - процесс образования опрокинутых складок, backwasting - уступание, basining - образование впадин, basking - нагревание на Солнце, оscillation - качание, bearing - отклонение, blocking - блокировка, boulis - обвал, boundage - граница, calving — процесс дробления, отделения, cementation цементация, peneplanation - выравнивание, petrification = fossilization - окаменение, plucking = sapping - ледниковое выпахивание , congelation - замерзание, congelifraction - дробление замораживанием, corrosion = corrosion - механическое воздействие обломочного материала на горные породы, ravinement оврагообразование, refusion - прочесс частичного расплавления горных пород, regelation - смерзание, removal - перемещение, replacement - замена, replenishing - пополнение, defluction - эластичное движение, deformation - прочесс, при котором породы слипаются в складки, degrade - разрушение, denudation - скрытие погребенных горных пород в результате выветривания, dероsition — отложение осадочных материалов, riving — растрескивание, desqua- 
mation - выветривание горных пород в результате шелушения, detrition - перетирание, diaparism - диапаризм, dilatancy - расширение, dislocation - перемещение, displacement - смещуение, sedimentation - отложение осадка, sedimentogenesis - образование осадка, dissection - расчленение, seepage фильтрация, distribution — распределение, separation = division - разделение, dolomitization - доломитизация (прочесс превращения известняка в доломит), shattering - раздробление, downwearing = flettering — выполаживание склонов, shifting - смещение, slumping - оползание, stabilization - закрепление, stoping обрушение, equiplanation = graviplanation - выравнивание, sublimination - поднятие наверх, superposion - напластование, evolution — эволючия, exhumation снятие покрова осадочных пород, swing - сдвиг, expansion — расширение, ехtrusion - выталкивание, falling - падание, fanglomerate - отложение конусов выноса, terracette - оползневая терраса, firnification - процесс превращчения снега в лед, thrusting - наступление, fissuring = fracturing - трещиноватость, transgression - интрузия, fluctiation - колебание, fluting - образование желобков, transition - переход, transportation - прочесс перемещения обломочной породь в другое место.

We think that the thesaurus representation of knowledge is an approximate explanation of the lexical system. Such a system makes it possible to observe the level organization of vocabulary and to determine the types of relations that would not have been explicitly expressed beyond the thesaurus scheme. These are key words and their role in the division of semantic fields.

\section{CONCLUSION}

Summing all up, it can be noted that the structure of the cognitive type of the scientific articles describing environmental problems, is a mental-linguistic frame used by the authors of the scientific articles in the process of generation of texts and the readers in the process of understanding. The structure of the cognitive type presents a scan of a specific topic and verbal fragments for each thematic unit. The reader is able to solve or suggest a solution to the critical environmental situation in the ecosystem based on the data obtained in the course of the text description of the environmental problems of mountain ecosystems. He can do it with a certain degree of inaccuracy because his personal picture of the world and of course his life experience are of great importance in fulfilling it. All information is presented in the given structure according to the principle of a thematic mini-dictionary, in which lexical filling of the topic blocks takes place. We hope that this structure and mini-thesaurus will serve as a basis for discursive activity.

In addition, we would like to note that the study of texts' corpus not only of articles on mountain ecosystems in Russian and English, but also in many other genres within the framework of any literature and any language will give new results for construction of cognitive type in different subject areas. 


\section{REFERENCES}

1. Baranov, A.G. (1999). Cognitive Formalisms of Text Activity. Pyatigorsk State University Bulletin, 2, 34-37. (In Russ.).

2. Baranov, A.G. (1993). Functional-pragmatic concept of text. Rostov: Rostov State University publ. (In Russ.).

3. Baranov, A.G. (2008). Pragmatics as the methodological perspective of the language. Krasnodar: Prosveschenie-Yug. publ. (In Russ.).

4. Karasik, V.I. (2002). Language circle: personality, concepts, discourse. Volgograd: Peremena publ. (In Russ.).

5. Lominina, Z.I. (2004). Emotional breadth of texts on ecology: trends and hypotheses. Ecological Bulletin of Research Centers of the Black sea Economic Cooperation (BSEC. Ecology of Language as Pragmatic Essence, 3, 45-48. (In Russ.).

6. Austin George. (1986). Word as action. New in foreign linguistics. Vol. XVII. The theory of speech acts. Moscow: Progress publ. pp. 22-129. (In Russ.).

7. Semenova, S.N. (2016). Compositional-Topical Modules of Content of Scientific-Popular Genre, Characterizing Subject Area "Geography" (on material of "National Geographic"). Bulletin of Peoples' Friendship University of Russia. Series: Theory of Language. Semiotics. Semantics, 2, pp. 56-61. (In Russ.).

8. Semenova, S.N. (2015). Cognitive Type of a Scientific Article on Ecological Problems of Mountain Ecosystems. Philological Sciences. Issues of Theory and Practice,10 (52), II. pp. 144-147. (In Russ.).

9. Searle, J.R. (1986). The classification of illocutionary acts. New in foreign linguistics, 17. Moscow: Progress publ. pp. 170-194. (In Russ.).

10. Abelson, R.P. (1981). Psychological Status of the Script Concept. American Psychologist, 36(7). pp. $715-729$.

11. Beaugrande, R. de. L. \& Dressler, W. (1994). Introduction to Text Linguistics. London: Longman publ.

12. Bellezza, F.S. \& Bower, G.H. (1982). Remembering script-based text. Poetics, 11(1), 1-23.

13. Dijk, T.A. van. (1995). Discourse Semantics and Ideology. Discourse and Society, 6(2), $243-$ 285.

14. Dijk, T.A. van. (1976). Pragmatics and Poetics. Pragmatics of Language and Literature, 2, $23-57$.

15. Divjak, D., Levshina, N. \& Klavan. J. (2016). Cognitive Linguistics: Looking Back, Looking Forward. Cognitive Linguistucs. 27(4), 447-463. DOI: 10.1515/cog-2016-0095.

16. Enquist, N.E. (1985). Stylistics, Text Linguistics and Composition. In Nordic Research in Text Linguistics and Discourse Analysis. 25-45.

17. Goral, M. \& Conner P.S. (2013). Language Disorders in Multilingual and Multicultural Populations. Annual Review of Applied Linguistics, 33, 128 - 161. DOI: 10.1017/S026719051300010x.

18. Gries, S.T. \& Ellis N.C. (2015). Statistical Measures for Usage-Based Linguistics. Language Learning. The Great Britain: John Wiley \& Sons, Inc publ., 65 (S1), pp. 228-255. DOI: 10.1111/lang.12119.

19. Jakobson, R. (1969). Linguistics in its Relation to other Sciences. Actes du $X^{e}$ congres international des linguists, 1. P. 76.

20. Krause, H., Bosch, S. \& Clahsen, H. (2014). Morphosyntax in the Bilingual Mental Lexicon: an Experimental Study of Strong Stems in German. Studies in Second Language Acquisition, 37(4), pp. 597-621. DOI: 10.1017/S02722623114000564.

21. Kruse, L. (1986). Drehbucher fur Verhaltensschauplatze oder: Skripts fur Settings. In: Ordnung and Variabilitat im Altagsgeschehen. pp. 135-153.

22. Lakoff, G. (1972). Language in Context. Language, 48(4), 907-927.

23. Mak, W.M., Tribushinina, E., \& Andreiushina, E. (2013). Semantics of Connectives Guides Referential Expectations in Discourse: an Eye-Tracking Study of Dutch and Russian. Discourse Processes, 50(8), 557-576. DOI: 10.1080/0163853x.2013.841075. 
24. Melinger, A., Branigan, H.P., \& Pickering, M.J. (2014). Parallel Processing in Language Production. Language, Cognition and Neuroscience, 29(6), 663-683. DOI: 10.1080/23273798.2014.906635.

25. Meutsch, D., \& Schmidt, S.J. (1986). On the Role of Conventions in Understanding Literary Texts. Amsterdam.

26. Nuyis, J. (2015). Subjectivity: Between Discourse and Conceptualization. Journal of Pragmatics, 86, pp. 106-110. DOI: 10.1016/j.pragma.2015.05.015.

27. Romanova, N. \& Gor, K. (2017). Processing of Gender and Number Agreement in Russian as a Second Language. Studies in Second Language Acquisition, 39(1). 97-128. DOI: $10.1017 / \mathrm{S} 0272263116000012$.

28. Schank, R.C. (1982). Reminding and memory organization: An introduction to MOPs. In: Strategies for natural language processing. Hillsdale (N.J.); L.: Erlbaum. pp. 455-493.

29. Stubbs, M. (1983). Discourse Analysis: The Sociolinguistic Analysis of Natural Language. Oxford.

30. Winke, P., Gass S., \& Sydorenko, T. (2013). Factors Influencing the Use of Captions by Foreign Learners: An Eye-Tracking Study. The Modern Language Journal, 97(1). 254-275. DOI: 10.1111/j.1540-4781.2013.01432.x.

\title{
Resourses
}

1. High Priorities. (2002). GEF's Contribution to Preserving and Sustaining Mountain Ecosystems. Washington, 13 (1). P. 30.

2. Meith, N. World Conservation: Mountain High. Switzerland, 2002. Vol. 33. № 1.

3. Mountain Momentum: Agenda for Today and Policy beyond IYM 2002. (2002). The United Nations University. 2002 International Year of Mountains. P. 26.

4. Nemolyaeva, L. (2001). Ensuring Long-Term Conservation of the Altai-Sayan Ecoregion WWF Project: Achievements and Lessons Learned. Moscow, P. 16.

5. Russian Bird Conservation Union. (1998). Moscow, P. 11.

6. Supporting Environmental Cooperation in Central Asia. (2002). Philippines, P. 49.

7. Tropical Montane Cloud Forests: Time for Action. (1999). Switzerland, P. 29.

\section{СТРУКТУРА КОГНИОТИПА НАУЧНОЙ СТАТЬИ О ГОРНЫХ ЭКОСИСТЕМАХ}

\author{
С.Н. Семенова \\ Кубанский государственный университет \\ ул. Ставропольская, 149, Краснодар, Россия, 350040
}

В статье представлена структура когниотипа научной статьи о горных экосистемах. Цель исследования - построить когниотип научной статьи, описывающей экологические проблемы горных экосистем. Для достижения поставленной в работе цели потребовалось решить следующие задачи: 1) изучить массив текстов научной статьи, описывающего горные экосистемы; 2) определить тематическую структуру когниотипа научной статьи, описывающего горные экосистемы; 3) представить речевые фрагменты к каждому композиционно-тематическому блоку; 4) описать прагматику текстовых реализаций научной статьи, описывающей горные экосистемы; 5) построить тезаурус слов и фраз. Автор обосновывает положение, что когниотип научной статьи - основная часть одного 
из когнитивных блоков в системе представления знаний человека в предметной области «Горные экосистемы». Выделены основные элементы: жизнедеятельность людей, загрязнение окружающей среды, охрана окружающей среды, постоянный мониторинг. В работе представлен ряд слов и словосочетаний, примеры которых расположены по субтемам на английском и русском языках. Данные слова и словосочетания служат выборкой из массива текстов предметной области «Горные экосистемы» и являются не готовыми текстами, а их заготовками для дискурсивной деятельности не только специалистов в этой области, но и всем тем, кто интересуется экологическими проблемами на английском и русском языках. Подводя итог, можно сказать, что структура когниотипа научной статьи - ментально-лингвистический фрейм, используемый авторами научных статей в процессе порождения текста и читателями в процессе понимания. Структура когниотипа представлена разверткой определенной темы и речевыми фрагментами к каждому тематическому блоку. Читатель способен решить или предложить выход из критической экологической ситуации, сложившейся в экосистеме основываясь на данные, полученные из текстового описания по экологическим проблемам горных экосистем.

Ключевые слова: структура когниотипа; предметная область «Горные экосистемы»; массив текстов; лингвистический фрейм; представление речевых фрагментов; дискурсивная деятельность; знания; когнитивная деятельность; прагматика текстовых реализаций; тезаурус

\section{БИБЛИОГРАФИЧЕСКИЙ СПИСОК}

1. Баранов А.Г. Когнитивные формализмы текстовой деятельности // Университетский вестник. 1999. № 2. С. 34-37.

2. Баранов А.Г. Функционально-прагматическая концепция текста. Ростов н/Д: Ростовский гос. ун-т, 1993.

3. Баранов А.Г. Прагматика как методологическая перспектива языка. Краснодар: Просвещение-Юг, 2008.

4. Карасик В.И. Языковой круг: личность, концепты, дискурс. Волгоград: Перемена, 2002.

5. Ломинина 3.И. Эмотивность текстов по экологии: тенденции и гипотезы // Экологический вестник научных центров ЧЭС. Экология языка как прагматическая сущность. 2004. C. $45-48$.

6. Остин Дж. Слово как действие // Новое в зарубежной лингвистике. Вып. XVII. Теория речевых актов. М.: Прогресс, 1986. С. 22-129.

7. Семенова С.Н. Композиционно-тематические блоки содержания жанра научно-популярной статьи, характеризующей предметную область «География» (на материале журнала "National Geopraphic") // Вестник Российского университета дружбы народов. Серия: Теория языка. Семиотика. Семантика. Москва, 2016. № 2. С. 56-61.

8. Семенова С.Н. Когниотип научной статьи об экологических проблемах горных экосистем // Филологические науки. Вопросы теории и практики. Тамбов, 2015. № 10 (52). Ч. II. C. $144-147$.

9. Серль Дж. Классификация иллокутивных актов // Новое в зарубежной лингвистике. Вып. 17. М.: Прогресс, 1986. С. 170-194.

10. Abelson R.P. Psychological Status of the Script Concept // American Psychologist. Vol. 36(7). pp. 715-729. Washington: APA Press, 1981.

11. Beaugrande R. de. L., Dressler W. Introduction to Text Linguistics. London: Longman, 1994.

12. Bellezza F.S., Bower G.H. Remembering script-based text// Poetics. 1982. Vol. 11. № 1. pp. $1-23$.

13. Dijk T.A. van. Discourse Semantics and Ideology // Discourse and Society. London, Thousands, Oaks, CA and New Delhi. 1995. Vol. 6. № 2. pp. 243-285.

14. Dijk T.A. van. Pragmatics and Poetics // Pragmatics of Language and Literature. Amsterdam: North-Holland Publishing Company, 1976. № 2. pp. 23-58.

15. Divjak D., Levshina N., Klavan J. Cognitive Linguistics: Looking Back, Looking Forward // Cognitive Linguistucs. Germany: Mourton de Gruyter, 2016. Vol. 27. № 4. Pp. 447-463. DOI: $10.1515 / \operatorname{cog}-2016-0095$. 
16. Enquist N.E. Stylistics, Text Linguistics and Composition // Nordic Research in Text Linguistics and Discourse Analysis. Trondheim, 1985. P. 25-45.

17. Goral M., Conner P.S. Language Disorders in Multilingual and Multicultural Populations // Annual Review of Applied Linguistics. Great Britain: Cambridge University Press. 2013. Vol. 33. pp. 128 - 161. DOI: 10.1017/S026719051300010x.

18. Gries S.T., Ellis N.C. Statistical Measures for Usage-Based Linguistics // Language Learning. The Great Britain: John Wiley \& Sons, Inc. 2015. Vol. 65. № S1. P. 228-255. DOI: 10.1111/lang.12119.

19. Jakobson R. Linguistics in its Relation to other Sciences // Actes du $\mathrm{X}^{\mathrm{e}}$ congres international des linguists. Bucarest, 1969. Vol. 1. p. 76.

20. Krause H., Bosch S., Clahsen H. Morphosyntax in the Bilingual Mental Lexicon: an Experimental Study of Strong Stems in German // Studies in Second Language Acquisition. The Great Britain: Cambridge University Press. 2014. Vol. 37. № 4. Pp. 597-621. DOI: $10.1017 / \mathrm{S} 02722623114000564$.

21. Kruse L. Drehbucher fur Verhaltensschauplatze oder: Skripts fur Settings // Ordnung and Variabilitat im Altagsgeschehen. Gottingen, 1986. P. 135-153.

22. Lakoff G. Language in Context // Language 48 (4). 1972. P. 907-927.

23. Mak W.M., Tribushinina E., Andreiushina E. Semantics of Connectives Guides Referential Expectations in Discourse: an Eye-Tracking Study of Dutch and Russian // Discourse Processes. The USA: Ablex Pub. Corp. 2013. Vol. 50, № 8. P. 557-576. DOI: 10.1080/0163853x.2013.841075.

24. Melinger A., Branigan H.P., Pickering M.J. Parallel Processing in Language Production // Language, Cognition and Neuroscience. The Great Britain: Routledge. 2014. Vol. 29. № 6. P. 663 -683. DOI: 10.1080/23273798.2014.906635.

25. Meutsch D., Schmidt S.J. On the Role of Conventions in Understanding Literary Texts. Amsterdam, 1986.

26. Nuyis J. Subjectivity: Between Discourse and Conceptualization // Journal of Pragmatics. The Netherlands: Elsevier Science Publishing Company, Inc. 2015. Vol. 86. P. 106-110. DOI: $10.1016 /$ j.pragma.2015.05.015.

27. Romanova N., Gor K. Processing of Gender and Number Agreement in Russian as a Second Language // Studies in Second Language Acquisition. The Great Britain: Cambridge University Press. 2017. Vol. 39. № 1. P. 97-128. DOI: 10.1017/S0272263116000012.

28. Schank R.C. Reminding and memory organization: An introduction to MOPs // Strategies for natural language processing. - Hillsdale (N.J.); L.: Erlbaum, 1982. P. 455- 493.

29. Stubbs M. Discourse Analysis: The Sociolinguistic Analysis of Natural Language. Oxford, 1983.

30. Winke P., Gass S., Sydorenko T. Factors Influencing the Use of Captions by Foreign Learners: An Eye-Tracking Study // The Modern Language Journal. The USA: John Wiley \& Sons, Inc. 2013. Vol. 97. № 1. P. 254-275. DOI: 10.1111/j.1540-4781.2013.01432.x.

\section{Источники}

1. High Priorities. GEF's Contribution to Preserving and Sustaining Mountain Ecosystems. Washington, 2002. Vol. 13. № 1. 2002.

2. Meith N. World Conservation: Mountain High. Switzerland, 2002. Vol. 33. № 1.

3. Mountain Momentum: Agenda for Today and Policy beyond IYM 2002. The United Nations University. 2002 International Year of Mountains. 2002.

4. Nemolyaeva L. Ensuring Long-Term Conservation of the Altai-Sayan Ecoregion WWF Project: Achievements and Lessons Learned. M., 2001.

5. Russian Bird Conservation Union. M., 1998.

6. Supporting Environmental Cooperation in Central Asia. Philippines, 2002.

7. Tropical Montane Cloud Forests: Time for Action. Switzerland, 1999. 


\section{Для цитирования:}

Семенова С.Н. Структура когниотипа научной статьи о горных экосистемах // Вестник Российского университета дружбы народов. Серия: Теория языка. Семиотика. Семантика, 2018. T. 9. № 2. C. 302-319. doi: 10.22363/2313-2299-2018-9-2-302-319.

\section{For citation:}

Semenova S.N. (2018). Structure of Cognitive Type of Scientific Article on Mountain Ecosystems. RUDN Journal of Language Studies, Semiotics and Semantics, 9 (2), 302-319. doi: 10.22363/23132299-2018-9-2-302-319.

Sofiya N. Semenova, 2018. RUDN Journal of Language Studies, Semiotics and Semantics, 9 (2), 302-319. doi: 10.22363/2313-2299-2018-9-2-302-319.

\section{Сведения об авторе:}

Семенова София Новиковна, кандидат филологических наук, доцент кафедры английского языка в профессиональной сфере факультета романо-германской филологии Кубанского государственного университета; научные интересы: социолингвистика, теория дискурса, семантика; e-mail: sofiya.semenova75@yandex.ru

\section{Bio Note:}

Semenova Sofiya Novikovna, Candidate of Philology, Associate Professor of English in Professional Sphere Department of Roman-German Faculty at the Kuban State University; Interests: sociolinguistics, theory of discourse, semantics; e-mail: sofiya.semenova75@yandex.ru 